\title{
CD73-Generated Adenosine: Orchestrating the Tumor-Stroma Interplay to Promote Cancer Growth
}

\author{
Bertrand Allard, Martin Turcotte, and John Stagg \\ Centre de Recherche, Centre Hospitalier, Faculté de Pharmacie l'Université de Montréal et Institut du Cancer de Montréal, \\ Montréal, QC, Canada H2L 4M1 \\ Correspondence should be addressed to John Stagg, john.stagg@umontreal.ca
}

Received 19 June 2012; Accepted 5 July 2012

Academic Editor: Karen M. Dwyer

Copyright ( $\odot 2012$ Bertrand Allard et al. This is an open access article distributed under the Creative Commons Attribution License, which permits unrestricted use, distribution, and reproduction in any medium, provided the original work is properly cited.

\begin{abstract}
Despite the coming of age of cancer immunotherapy, clinical benefits are still modest. An important barrier to successful cancer immunotherapy is that tumors employ a number of mechanisms to facilitate immune escape, including the production of antiinflammatory cytokines, the recruitment of regulatory immune subsets, and the production of immunosuppressive metabolites. Significant therapeutic opportunity exists in targeting these immunosuppressive pathways. One such immunosuppressive pathway is the production of extracellular adenosine by CD73, an ectonucleotidase overexpressed in various types of cancer. We hereafter review the biology of CD73 and its role in cancer progression and metastasis. We describe the role of extracellular adenosine in promoting tumor growth through paracrine and autocrine action on tumor cells, endothelial cells, and immune cells.
\end{abstract}

\section{Cancer Immunotherapy: An Overview}

The recent FDA approval of ipilimumab (Yervoy, BristolMyers Squibb)—an antibody that blocks the inhibitory $\mathrm{T}$ cell receptor CTLA-4-for treatment of metastatic melanoma and sipuleucel-T (Provenge, Dendreon)—a cellbased vaccine-for treatment of castration-resistant prostate cancer, has revitalized the interest for cancer immunotherapy [1]. The enthusiasm generated by these new treatments is further fuelled by overwhelming new data revealing the importance of tumor immune infiltrates in the survival of cancer patients. Indeed, the presence of $\mathrm{CD} 8+\mathrm{CD} 45 \mathrm{RO}+\mathrm{T}$ cells in tumors is associated with a good prognosis in various types of epithelial cancers [2]. In cancers such as colorectal cancers, T-cell infiltration has in fact superior prognostic power than standard staging methods [3].

In addition of being involved in the natural progression of cancer, immune responses affect the activity of anticancer treatments [4]. Accordingly, recent studies revealed that some chemotherapeutic drugs, such as anthracyclines and oxaliplatin, specifically rely on the induction of anticancer immune responses for therapeutic activity [5]. Immune responses also play a major role in the efficacy of targeted therapies with monoclonal antibodies (mAbs). While antibody-dependent cellular cytotoxicity (ADCC) is important in the activity of tumor-targeted $\mathrm{mAb}$ therapies, recent studies suggest that mAbs such as trastuzumab may also stimulate adaptive antitumor immunity [6]. Taken together, this suggests that incorporating immunotherapeutic approaches to standard treatments might in fact be synergistic.

Much of the recent successes in cancer immunotherapy come from blocking mAbs targeting immune checkpoint inhibitors, such as CTLA-4 and PD-1. In 2011, the FDA approved the use of the anti-CTLA- 4 mAb ipilimumab in patients with metastatic melanoma. However, one of the drawbacks to anti-CTLA- $4 \mathrm{mAb}$ therapy is the generation of autoimmune toxicities due to on-target effects. Accordingly, it has been reported that up to $23 \%$ of patients treated with ipilimumab developed serious grade 3-4 adverse events [7]. Another promising form of cancer immunotherapy consists of blocking mAbs against PD-1 or its ligand PD-L1. Administration of anti-PD-1 or anti-PD-L1 mAb enhances adaptive anti-tumor immune responses by preventing $\mathrm{T}$ cell exhaustion. In early clinical trials, both anti-PD-1 and anti-PD-L1 mAbs have shown impressive objective responses 


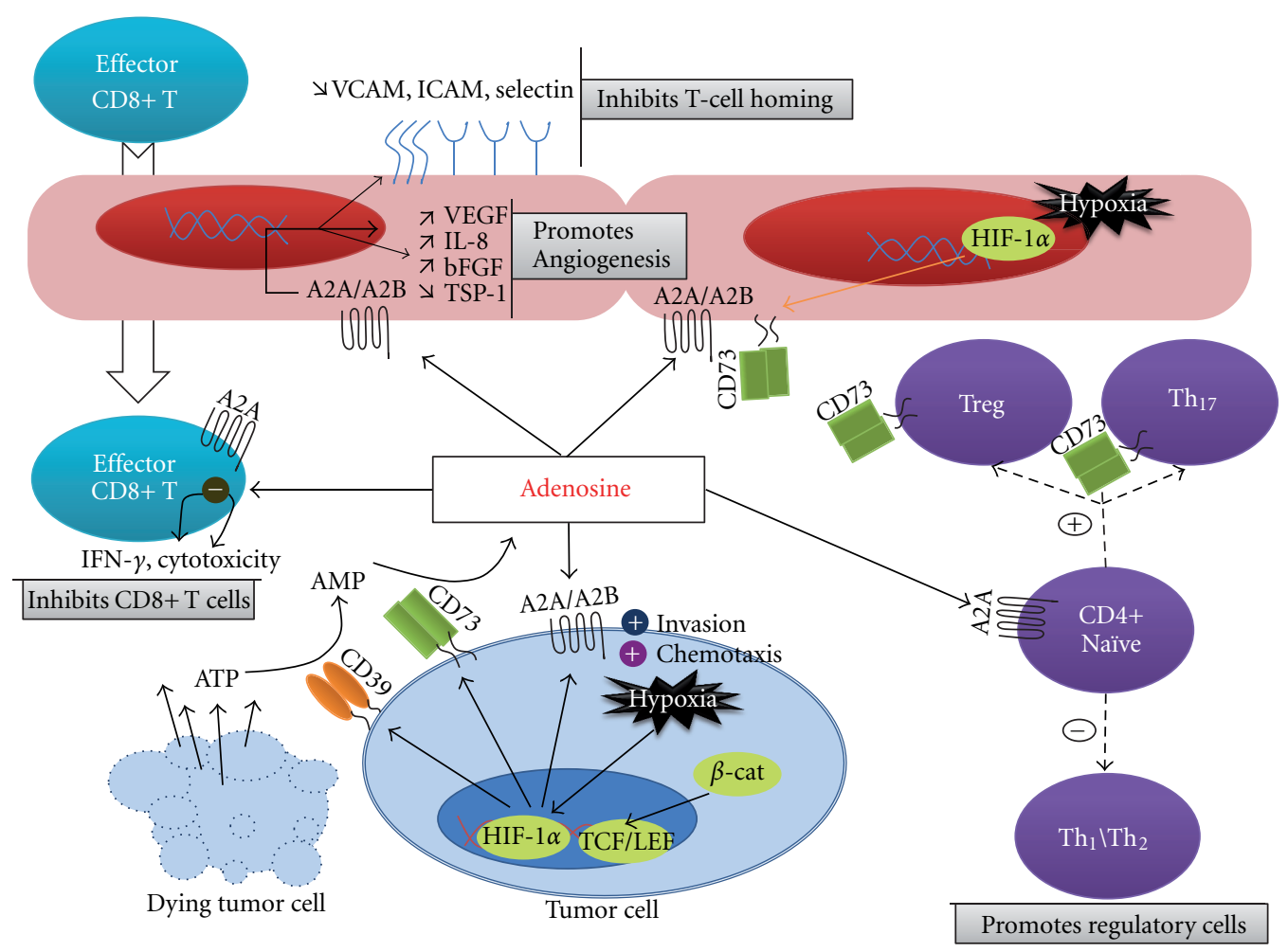

FIGURE 1: CD73-generated adenosine orchestrates the tumor-stroma interplay to promote cancer growth. The concerted action of CD39 and CD73 represents the main pathway for extracellular adenosine production in the tumor microenvironment. These two ectonucleotidases are expressed not only by tumor stromal cells (such as endothelial cells or tumor-associated regulatory $\mathrm{T}$ cells) but also by certain cancer cells, allowing for the conversion of extracellular ATP (released by dying tumor cells) into adenosine. Adenosine exerts its tumor-promoting effects in paracrine and autocrine fashion by activating adenosine receptors expressed by tumors cells, endothelial cells, or immune cells. Activation of A2A adenosine receptors inhibits IFN- $\gamma$ production and cytotoxic killing by CD8+ T cells and promotes CD4+ cells differentiation into T-regulatory cells. This immunosupressive effect is strengthened by adenosine action on the tumor-surrounding endothelium which consists in repressing T-cell homing to tumors through the downmodulation of adhesion proteins such as ICAM-1, VCAM-1 or P-selectin. Simultaneously, A2A and A2B engagement on endothelial cells also enhance the production of proangiogenic factors including VEGF, b-FGF, and IL-8. This effect is mediated by HIF-1 and synergizes with the hypoxic tumoral microenvironment. Finally, CD73-generated adenosine also promotes tumor development by directly acting on cancer cells through $\mathrm{A} 2 \mathrm{~A}$ and/or $\mathrm{A} 2 \mathrm{~B}$ adenosine receptor activation and subsequent enhancement of invasiveness and chemotactic response.

in patients with nonsmall-cell lung cancer, melanoma, and renal-cell cancer $[8,9]$.

Despite this coming of age of cancer immunotherapy, clinical benefits are still modest. One potential explanation is that tumors employ a number of mechanisms to facilitate immune escape, including the production of anti-inflammatory cytokines, the recruitment of regulatory immune subsets, and the production of immunosuppressive metabolites. Significant therapeutic opportunity exists in targeting these immunosuppressive pathways. One such therapeutic target is $\mathrm{CD} 73$, an ectoenzyme that catalyses the generation of extracellular adenosine, a potent immunosuppressive molecule. We hereafter review the biology of CD73 and its role in cancer progression and metastasis.

\section{CD73 Biology and the Adenosinergic Signaling}

CD73 is a glycosylphosphatidylinositol (GPI-) anchored nucleotidase present in cell membrane lipid rafts, active as a disulfide-linked homodimer, which catalyses the hydrolysis of extracellular adenosine monophosphate (AMP) into adenosine [10]. CD73 is expressed on lymphocytes, endothelial and epithelial cells, where it participates in ion transport regulation, endothelial cell barrier function, endothelial homeostasis, and protection from ischaemia [11-13].

CD73 also has a predominant role in immunity (Figure 1). Indeed, CD73 negatively regulates the proinflammatory effects of extracellular adenosine triphosphate (ATP). Extracellular ATP, released by damaged or dying cells and bacteria, promotes the recruitment of immune phagocytes [14] and activates P2X7R, a coactivator of the NLRP3 inflammasome, which then triggers the production of proinflammatory cytokines, such as IL-1 $\beta$ and IL-18 [15]. The catabolism of extracellular ATP into ADP, AMP and adenosine is controlled by ectonucleotidases and membranebound kinases. Whilst hydrolysis of ATP into AMP is predominantly performed by CD39 (ENTPD1), CD73 catalyses the conversion of AMP into adenosine. Hence, CD39 and CD73 act in concert to convert proinflammatory ATP into 
immunosuppressive adenosine. Importantly, the activity of CD39 is reversible by the actions of NDP kinase and adenylate kinase, whereas the activity of CD73 is virtually irreversible. Thus, CD73 represents a crucial checkpoint in the conversion of proinflammatory ATP into immunosuppressive adenosine.

To mediate its physiological actions, CD73-generated adenosine can bind to four distinct G-protein-coupled receptors: $\mathrm{A} 1, \mathrm{~A} 2 \mathrm{~A}, \mathrm{~A} 2 \mathrm{~B}$, and $\mathrm{A} 3$ [16]. The $\mathrm{A} 1$ and $\mathrm{A} 3$ adenosine receptors are coupled with the $\mathrm{G}_{\mathrm{i} / \mathrm{o}}$ subunit, which leads to the inhibition of adenylyl cyclase, cyclic AMP (cAMP) production, and protein kinase A (PKA) activation. A1 and A3 receptors have also been linked to the activation of the phosphatidylinositol 3-kinase (PI3 K) pathway. In contrast, $A 2 A$ and $A 2 B$ are coupled with the $G_{s}$ subunit that stimulates cAMP production, and PKA activation. All four adenosine receptors have been associated with the activation of the mitogen-activated protein kinase (MAPK) and protein kinase C (PKC) pathways.

\section{Expression of CD73 on Cancer Cells}

CD73 has been found to be overexpressed in several types of cancer, including bladder cancer, leukemia, glioma, glioblastoma, melanoma, ovarian cancer, colon cancer, and breast cancer [17-20]. This overexpression can be explained by several mechanisms. In the context of breast cancer, loss of estrogen receptor (ER) expression has been shown to induce constitutive CD73 expression [20]. Thus, CD73 is highly expressed in ER-negative breast cancer cells and might constitute a promising target for treatment-refractory breast tumors such as ER-negative or triple-negative breast cancer [21].

Another factor that can drive CD73 expression is the hypoxic nature of the tumor microenvironment. Indeed, the CD73 gene promoter has at least one binding site for the hypoxia-inducible factor (HIF-) $1 \alpha$ and, not surprisingly, hypoxia promptly stimulates CD73 expression. Inhibition of HIF- $1 \alpha$ by antisense oligonucleotides or point mutations in the hypoxia response element of the CD73 promoter has been reported to inhibit hypoxia-induced CD73 expression [22-24]. Notably, hypoxia also upregulates CD39, A2A, and $\mathrm{A} 2 \mathrm{~B}$ adenosine receptor expression $[25,26]$.

Another pathway that can induce CD73 expression is the Wnt pathway. Accordingly, a TCF/LEF consensus binding site is present in the CD73 gene promoter and it has been shown that Wnt signaling can drive CD73 expression [27, 28]. Of interest, the Wnt pathway is often deregulated in human tumors by the loss of the tumor suppressor APC or by mutations in the $\beta$-catenin gene [29].

CD73 expression is also regulated epigenetically. Wang et al. recently reported that CD73 expression is downregulated by methylation-dependent transcriptional silencing in several human melanoma cell lines [30]. Notably, relapse with metastatic disease was found to be more frequent in melanoma patients lacking CD73 methylation. While this observation must be validated in a larger cohort, it supports the notion that CD73 might constitute a valid therapeutic target in melanoma.
Finally, CD73 overexpression on tumors may also result from a selective pressure exerted by the immune system. Indeed CD73 positive tumor cells, via the production of immunosuppressive adenosine, are better equipped to evade anti-tumor immune responses.

\section{Prognostic Implications of CD73 Expression}

Despite the fact that CD73 expression has been observed in several types of cancer, correlative analysis to clinical outcome has been relatively limited [31]. Recently, Wu et al. evaluated the prognostic relevance of CD73 in colorectal cancer (CRC): analysis of CD73 expression by immunohistochemistry (IHC) revealed that high levels of CD73 were correlated with a poor prognosis ( $n=342$ patients) [32]. In breast cancer, one study suggested a negative correlation between ER and CD73 expression ( $n=18$ patients) [20] and a retrospective analysis of 30 breast cancer biopsies found that CD73 expression was associated with an increased risk of relapse and increased likelihood of metastasis [33]. In contrast, another recent study reported that high CD73 expression levels were associated with a good prognosis in breast cancer patients [34]. The prognostic implication of CD73 expression in breast cancer thus remains controversial.

A recent study assessed the clinical implication of CD73 in chronic lymphoblastic leukemia (CLL) [17]. The study evaluated CD39 and CD73 expression on 299 blood samples and found that all CLL samples expressed CD39 while only one third expressed CD73. CD73 expression was found to be highest on CD38+, ZAP-70+, or Ki-67+ leukemic cells, suggesting that $\mathrm{CD} 73$ was associated with a more aggressive and proliferative disease. CD73 was found to be particularly abundant in lymph nodes, on highly proliferating cells and in perivascular areas suggesting a role in lymph node homing. The authors also identified a significant elevation of A2A adenosine receptor expression in leukemic patients compared to healthy controls. Notably, A2A activation on CLL cells was linked to an increase resistance to drug-induced apoptosis. Taken together, these results suggest that adenosine is part of an autocrine/paracrine loop that enhances CLL cells chemoresistance and favours their arrest in lymph node proliferation centers [17].

\section{CD73 Expression on Regulatory T Cells}

T-regulatory cells (Tregs) are naturally occurring or inducible $\mathrm{T}$ cells specialized in suppression of immune responses. Treg-mediated immunosuppression can operate through several mechanisms, including CD73-mediated production of extracellular adenosine, which has recently emerged as a key process implemented by Tregs and exploited by various tumors to dampen immune responses [31].

CD73 is expressed on different subsets of T lymphocytes, but it is particularly abundant in Foxp3+ Tregs (37). In mice, CD73 is expressed in approximately $60 \%$ of $\mathrm{CD} 4+$ and $80 \%$ of CD8+ $\mathrm{T}$ cells and is predominant in CD25high/CD39+/Foxp3+ T cells [35-37]. The coexpression of CD73 and CD39 on Treg provides them with the complete enzymatic machinery to produce immunosuppressive 
adenosine. In humans, CD73 expression in T cells is more limited, with CD73 expression highly correlated to the presence of CD25, CD39, and Foxp3 on CD4 T cells [38]. Amongst human Tregs, 70-80\% coexpresses CD73 and CD39 [38]. In human Tregs, CD73 appears to be stored intracellularly [38], which may account for previous reports pointing out to a large discrepancy concerning CD73 expression in mouse and human Tregs $[39,40]$. CD73 expression on Tregs is modulated upon activation and depends on the cytokine milieu. Indeed, it has been shown that CD73 expression is augmented upon TCR engagement concomitantly to $\mathrm{P} 2 \mathrm{X} 7$ receptor downmodulation, thus favoring the production of immunosuppresive adenosine and limiting proapoptotic effects of ATP [41]. Another study has demonstrated that CD73 expression is enhanced when CD4+ cells are activated in the presence of TGF- $\beta$ [36]. This effect has been observed in both CD4+/Foxp3+ and CD4+/Foxp3- cells, confirming that CD73 upregulation can occur independently of Foxp3. A recent publication confirmed these results and showed that both TGF- $\beta$ and IL-6 are required for CD73 and CD39 expression on Th17 cells [42]. Signaling pathways investigation revealed that TGF- $\beta$-induced inhibition of Gfi1 transcription repressor coupled to IL-6-induced STAT-3 activation was necessary for ectonucleotidase expression on Th17 cells. In several murine cancer models where TGF$\beta$ accumulates in the tumor microenvironment, CD39 and CD73 expression on Th17 cells actively participates in tumor immunoevasion [42].

CD73-mediated production of adenosine is thus crucial for Tregs immunosuppressive potential [35]. This has been revealed by an impaired immunosuppressive potential of Tregs derived from CD39- or CD73-deficient mice [35, 41]. In CD73-deficient mice, activated CD4+ cells showed an augmented production of proinflammatory cytokines (IFN$\gamma$, IL-2, and TNF- $\alpha$ ) [41] and loss of immunosuppressive function of Tregs. In CD39-deficient mice, Tregs were shown to be constitutively activated, thus abrogating their suppressive activity on non-Tregs cells [35]. Taken together, these studies demonstrated that Tregs immunosuppression relies, at least in part, on the hydrolysis of extracellular ATP into adenosine by the concerted action of CD39 and CD73 and the subsequent engagement of A2A on effector T cells. A2A activation on T-effector cells induces a long-term anergy, characterized by an impaired proliferation upon TCR engagement and a reduction in proinflammatory cytokines production [43]. Moreover, this anergy-like state cannot be reversed by TCR reengagement, even in the absence of A2A signaling. Complementary in vivo experiments, using a model of lung autoimmunity, showed that A2A activation is involved in Th17 cells inhibition (via the inhibition of IL-6 synthesis) and in the induction of immunosuppressive LAG3+ Treg cells [43].

The importance of CD73 expression on Tregs has also been documented in various tumor models [44, 45]. Using DEREG transgenic mice (expressing the diphtheria toxin receptor under the control of Foxp3) adoptively reconstituted with Tregs from CD73-deficient or wild type mice, Stagg et al. demonstrated that CD73 expression on Tregs is crucial to promote the growth of MC38 colon tumors [44].
Another independent research group obtained similar results with B16F10 tumors implanted in RAG1-/- mice adoptively transferred with CD73-deficient or wild type T cells, with or without depletion of CD25+ T cells $[45,46]$. These studies highlighting the role of CD73 on mouse Tregs have been corroborated by observations made in human Tregs. Accordingly, analysis of PBMC of cancer patients revealed that the expression and frequency of CD73+ Tregs are elevated compared to healthy volunteers [47]. Moreover, Tregs obtained from cancerous patients have increased nucleotidase activity, increased suppressor functions, and are able to infiltrate tumors [47]. Interestingly, complementary in vitro studies pointed out to a synergistic effect of adenosine and prostaglandin E2 (PGE2) in mediating Treg suppression [48]. Notably, COX2+ tumor cells were demonstrated to promote adenosine and PGE2 synthesis by Tregs in coculture experiments.

\section{CD73 Expression on Endothelial Cells}

Vascular and lymphatic endotheliums are crucial organs for leukocytes trafficking into tissues. The selective permeability and barrier function of the endothelium are tightly controlled and dysregulation of endothelial barrier hemostasis is associated with several diseases, including cancer.

CD73 and adenosine receptors are important regulators of leucocyte trafficking and endothelial hemostasis [49]. CD73 possesses distinct roles on lymphatic and vascular endothelium [50]. On vascular endothelial cells, CD73 participates in leukocytes extravasation from blood. It has been shown that leukocyte binding on endothelial cell triggers the inhibition of CD73 nucleotidase activity, thereby reducing local adenosine production which favors vascular permeability and leukocyte transmigration [12]. Leukocyte CD73 instead contributes to trafficking across lymphatics [50]. The importance of CD73 on endothelial cell function has also been largely evidenced by the analysis of CD73 deficient mice [51]. CD73-deficient mice present elevated expression of VCAM-1 in carotid arteries associated with an increased accumulation of monocytes [52]. Unexpectedly, in experimental autoimmune encephalomyelitis (EAE, a model of multiple sclerosis), CD73-deficiency resulted in resistance to the disease [53]. This resistance is thought to be the result of a lack of lymphocyte infiltration in the central nervous system. Interestingly, A2A blockade protected wild type mice against EAE and was associated with a downregulation of ICAM-1 expression on the choroid plexus.

In humans, a recent study identified mutations in the CD73 gene resulting in a nonfunctional protein and the development of symptomatic arterial and joint calcification, a pathology associated with an excess risk of cardiovascular events. The increase in ectopic tissue calcification associated with a non-functional CD73 protein was found to be dependent on an increase in tissue nonspecific alkaline phosphatase (TNAP). Therefore, targeted blockade of CD73 as a therapeutic approach could theoretically be combined with inhibitors of TNAP such as bisphosphonates or lansoprazole in order to prevent the risk of arterial calcification [54]. 
Activation of the adenosinergic system on endothelial cells is largely regulated by hypoxia. A2B adenosine receptor and neutrophils play a central role in promoting endothelial cell barrier function during hypoxia $[25,55$, 56]. Hypoxia upregulates A2B expression via HIF- $1 \alpha$ [57] and activation of $\mathrm{A} 2 \mathrm{~B}$ potentiates the secretion of VEGF and other proangiogenic factors such as IL-8 [58] or basic fibroblast growth factor (bFGF) [59]. Hypoxia further drives expression of A2A via HIF-2 $\alpha$. A2A activation in endothelial cells has been to shown enhance proliferation, migration, and capillary-like tube formation [60]. Interestingly, A2Amediated tube formation was shown to depend on the inhibition of the antiangiogenic factor thrombospondin1 [61]. Adenosine receptor activation on endothelial cells was also demonstrated to enhance VE-cadherin expression in a $\mathrm{A} 2 \mathrm{~A}$, dependent manner, $\mathrm{P}$-selectin expression via $\mathrm{A} 2 \mathrm{~B}$ and $\mathrm{CD} 73$ expression in a $\mathrm{A} 2 \mathrm{~B}$ dependent fashion $[58,62,63]$.

In addition to hypoxia, CD73 and adenosine receptors have been shown to be regulated by cytokines and growth factors. For instance, types I and II IFNs increase CD73 expression at the surface of endothelial cells, thus promoting adenosine generation and subsequent enhancement of endothelial cell barrier function $[64,65]$. Likewise, A2A and A2B expressions are augmented on endothelial cells upon IL-1 or TNF- $\alpha$ stimulation [66]. Surprisingly, TNF- $\alpha$ does not upregulate CD73 but, on the contrary, reduces CD73 nucleotidase activity by triggering CD73 shedding from plasma membrane in a PLC-dependent manner [67]. Interestingly, CD73 shedding has been described on lymphocytes upon CD73 engagement by a specific monoclonal antibody and was associated with enhanced lymphocyte adhesion to endothelial cells through a calpain-like enzyme-mediated LFA-I clustering $[68,69]$. In contrast, endothelial CD73 engagement does not entail its cleavage from plasma membrane.

\section{CD73 and Tumor Metastasis}

Tumor metastasis is a complex multistep process associated with poor prognosis [70]. Therefore, identification of metastasis-promoting pathways is of primary importance for the development of new anticancer treatments. In this regard, accumulating data indicate that CD73 promotes tumor metastasis. Two independent studies have correlated CD73 expression with lymph node metastasis of breast tumors [33, 71]. In both studies, relevance of the results obtained with cell lines and murine models was confirmed in patient biopsies, revealing that CD73 is upregulated in metastatic tumors, lymph node foci and associated with disease relapse [33]. CD73 upregulation in highly metastatic breast tumor cells might be associated with a loss of $\mathrm{CpG}$ island methylation in the NT5E gene, as recently observed by Wang et al. [30].

CD73 implication in tumor metastasis has been evidenced in our recent studies using CD73-null mice. We showed that CD73-deficient mice are resistant to experimental lung metastasis following intravenous injection of
B16F10 melanoma cells or TRAMP-C1 prostate cancer cells $[44,72]$. Interestingly, the resistance of CD73 $\mathrm{KO}$ mice to experimental metastasis was independent of the immune system. This observation infers that CD73 expression on nonhematopoietic cells, presumably endothelial cells, promotes the metastatic process. CD73 expression on tumor cells can also promote tumor metastasis in mice, most likely via an autocrine activation of $\mathrm{A} 2 \mathrm{~B}$ adenosine receptor [73]. This is concordant with data obtained by other groups who reported that CD73 overexpression or adenosine receptors activation on cancer cells can promote chemotaxis and invasiveness [74-78].

\section{CD73 Targeting for Cancer Treatment}

Currently, accumulating preclinical data provided by our laboratory and others underscore the therapeutic potential of CD73 blockade for cancer therapy. The analysis of tumorigenesis in CD73-null mice revealed that a lack of CD73 expression can efficiently delay tumors growth and confer metastasis resistance in a variety of murine tumor models $[44-46,72]$. All these studies converge on the fact that CD73 $\mathrm{KO}$ mice are protected against experimental tumorigenesis because of the absence of Treg-generated adenosine [44, 46]. When CD73 is also expressed on tumor cells, blockade of CD73 on both host and tumor cells is required to achieve optimal antitumor effect $[45,72]$. It should be noted that experimental tumorigenesis is also delayed in CD39- or A2A-null mice $[79,80]$, thus supporting the notion that the adenosinergic system is a relevant target for cancer therapy.

\section{Conclusion}

CD73 inhibition with $\alpha, \beta$-methylene adenosine diphosphate (APCP) or neutralizing monoclonal antibodies $(\mathrm{mAb})$ have demonstrated antitumor effects in various tumor models $[73,81,82]$. Notably, combination of adoptive T-cell therapy with CD73 blockade was shown to be synergistic [45, 81]. This successful combination paves the way for the investigation of other bi- or multitherapies combining CD73 inhibition with immune activating agents (e.g., anti-PD1, anti-CTLA-4, and anti-TIM-3). CD73 blockade could also be combined with proimmunogenic chemotherapeutic drugs $[83,84]$. Apart from their effect on tumor immunity, CD73 antagonists also have the potential to directly inhibit autocrine protumorigenic effects of CD73 on tumor cells, such as increased migration or survival. The use of neutralizing $\mathrm{mAbs}$ could be of particular interest as their antagonist properties on CD73 could potentiate their intrinsic immunemediated cytotoxic activities through the engagement of $\mathrm{F} c \gamma \mathrm{R}$ on immune cells. Taken together, these observations suggest that CD73 is a promising therapeutic target for the treatment of various cancers. In particular, association of CD73 blockade with other classic or newly developed anticancer agents seems extremely attractive. Future studies aiming at translating these results into therapeutic benefit for patients are warranted. 


\section{Acknowledgments}

J. Stagg is supported by research grants from the Canadian Institutes of Health Research and the Cancer Research Society, Canada. The authors do not have any conflict of interests with the content of the paper.

\section{References}

[1] I. Mellman, G. Coukos, and G. Dranoff, "Cancer immunotherapy comes of age," Nature, vol. 480, no. 7378, pp. 480-489, 2011.

[2] J. Galon, F. Pagès, F. M. Marincola et al., "The immune score as a new possible approach for the classification of cancer," Journal of Translational Medicine, vol. 10, article 1, 2012.

[3] B. Mlecnik, M. Tosolini, A. Kirilovsky et al., "Histopathologicbased prognostic factors of colorectal cancers are associated with the state of the local immune reaction," Journal of Clinical Oncology, vol. 29, no. 6, pp. 610-618, 2011.

[4] L. Galluzzi, L. Senovilla, L. Zitvogel, and G. Kroemer, "The secret ally: immunostimulation by anticancer drugs," Nature Reviews Drug Discovery, vol. 11, no. 3, pp. 215-233, 2012.

[5] Y. Ma, L. Aymeric, C. Locher et al., "Contribution of IL-17producing $\gamma \delta$ T cells to the efficacy of anticancer chemotherapy," Journal of Experimental Medicine, vol. 208, no. 3, pp. 491503, 2011.

[6] J. Stagg, S. Loi, U. Divisekera et al., "Anti-ErbB-2 mAb therapy requires type I and II interferons and synergizes with anti-PD1 or anti-CD137 mAb therapy," Proceedings of the National Academy of Sciences of the United States of America, vol. 108, no. 17, pp. 7142-7147, 2011.

[7] C. Robert, L. Thomas, I. Bondarenko et al., "Ipilimumab plus dacarbazine for previously untreated metastatic melanoma," New England Journal of Medicine, vol. 364, no. 26, pp. 25172526, 2011.

[8] S. L. Topalian, F. S. Hodi, J. R. Brahmer et al., "Safety, activity, and immune correlates of anti-PD-1 antibody in cancer," New England Journal of Medicine, vol. 366, no. 26, pp. 2443-2454, 2012.

[9] J. R. Brahmer, S. S. Tykodi, L. Q. M. Chow et al., "Safety and activity of anti-PD-L1 antibody in patients with advanced cancer," New England Journal of Medicine, vol. 366, no. 26, pp. 2455-2465, 2012.

[10] J. Stagg and M. J. Smyth, "Extracellular adenosine triphosphate and adenosine in cancer," Oncogene, vol. 29, no. 39, pp. 5346-5358, 2010.

[11] D. G. Shirley, R. M. Vekaria, and J. Sévigny, "Ectonucleotidases in the kidney," Purinergic Signalling, vol. 5, no. 4, pp. 501-511, 2009.

[12] T. Henttinen, S. Jalkanen, and G. G. Yegutkin, "Adherent leukocytes prevent adenosine formation and impair endothelial barrier function by ecto- $5^{\prime}$-nucleotidase/CD73-dependent mechanism," Journal of Biological Chemistry, vol. 278, no. 27, pp. 24888-24895, 2003.

[13] T. Eckle, T. Krahn, A. Grenz et al., "Cardioprotection by ecto-5' -nucleotidase (CD73) and A2B adenosine receptors," Circulation, vol. 115, no. 12, pp. 1581-1590, 2007.

[14] M. R. Elliott, F. B. Chekeni, P. C. Trampont et al., "Nucleotides released by apoptotic cells act as a find-me signal to promote phagocytic clearance," Nature, vol. 461, no. 7261, pp. 282-286, 2009.

[15] L. Zitvogel, O. Kepp, L. Galluzzi, and G. Kroemer, "Inflammasomes in carcinogenesis and anticancer immune responses," Nature Immunology, vol. 13, no. 4, pp. 343-351, 2012.
[16] B. B. Fredholm, A. P. IJzerman, K. A. Jacobson, J. Linden, and C. E. Müller, "International union of basic and clinical pharmacology. LXXXI. Nomenclature and classification of adenosine receptors-an update," Pharmacological Reviews, vol. 63, no. 1, pp. 1-34, 2011.

[17] S. Serra, A. L. Horenstein, T. Vaisitti et al., "CD73-generated extracellular adenosine in chronic lymphocytic leukemia creates local conditions counteracting drug-induced cell death," Blood, vol. 118, no. 23, pp. 6141-6152, 2011.

[18] L. Bavaresco, A. Bernardi, E. Braganhol et al., "The role of ecto-5' nucleotidase/CD73 in glioma cell line proliferation," Molecular and Cellular Biochemistry, vol. 319, no. 1-2, pp. 6168, 2008.

[19] R. Sadej, J. Spychala, and A. C. Skladanowski, "Expression of ecto-5' -nucleotidase (eN, CD73) in cell lines from various stages of human melanoma," Melanoma Research, vol. 16, no. 3, pp. 213-222, 2006.

[20] J. Spychala, E. Lazarowski, A. Ostapkowicz, L. H. Ayscue, A. Jin, and B. S. Mitchell, "Role of estrogen receptor in the regulation of ecto- $5^{\prime}$-nucleotidase and adenosine in breast cancer," Clinical Cancer Research, vol. 10, no. 2, pp. 708-717, 2004.

[21] M. J. Haas, "CD73: double-breasted suit, SciBX: ScienceBusiness eXchange 3," 2010.

[22] K. Synnestvedt, G. T. Furuta, K. M. Comerford et al., "Ecto-5' nucleotidase (CD73) regulation by hypoxia-inducible factor-1 mediates permeability changes in intestinal epithelia," Journal of Clinical Investigation, vol. 110, no. 7, pp. 993-1002, 2002.

[23] X. Li, T. Zhou, X. Zhi, F. Zhao, L. Yin, and P. Zhou, "Effect of hypoxia/reoxygenation on CD73 (ecto-5'-nucleotidase) in mouse microvessel endothelial cell lines," Microvascular Research, vol. 72, no. 1-2, pp. 48-53, 2006.

[24] G. L. Semenza, "Hypoxia-inducible factor 1: regulator of mitochondrial metabolism and mediator of ischemic preconditioning," Biochimica et Biophysica Acta, vol. 1813, no. 7, pp. 1263-1268, 2011.

[25] H. K. Eltzschig, J. C. Ibla, G. T. Furuta et al., "Coordinated adenine nucleotide phosphohydrolysis and nucleoside signaling in posthypoxic endotheliumml: role of ectonucleotidases and adenosine A 2B receptors," Journal of Experimental Medicine, vol. 198, no. 5, pp. 783-796, 2003.

[26] H. K. Eltzschig, D. Kö hler, T. Eckle, T. Kong, S. C. Robson, and S. P. Colgan, "Central role of Sp1-regulated CD39 in hypoxia/ischemia protection," Blood, vol. 113, no. 1, pp. 224232, 2009.

[27] J. Spychala, A. G. Zimmermann, and B. S. Mitchell, "Tissuespecific regulation of the ecto- $5^{\prime}$-nucleotidase promoter. Role of the cAMP response element site in mediating repression by the upstream regulatory region," Journal of Biological Chemistry, vol. 274, no. 32, pp. 22705-22712, 1999.

[28] J. Spychala and J. Kitajewski, "Wnt and $\beta$-catenin signaling target the expression of ecto- 5 '-nucleotidase and increase extracellular adenosine generation," Experimental Cell Research, vol. 296, no. 2, pp. 99-108, 2004.

[29] P. Polakis, "Wnt signaling and cancer," Genes and Development, vol. 14, no. 15, pp. 1837-1851, 2000.

[30] H. Wang, S. Lee, C. Lo Nigro et al., "NT5E (CD73) is epigenetically regulated in malignant melanoma and associated with metastatic site specificity," British Journal of Cancer, vol. 106, no. 8, pp. 1446-1452, 2012.

[31] P. A. Beavis, J. Stagg, P. K. Darcy, and M. J. Smyth, "CD73: a potent suppressor of antitumor immune responses," Trends in Immunology, vol. 33, no. 5, pp. 231-237, 2012. 
[32] X.-R. Wu, X.-S. He, Y.-F. Chen et al., "High expression of CD73 as a poor prognostic biomarker in human colorectal cancer," Journal of Surgical Oncology, vol. 106, no. 2, pp. 130137, 2012.

[33] R. Leth-Larsen, R. Lund, H. V. Hansen et al., "Metastasisrelated plasma membrane proteins of human breast cancer cells identified by comparative quantitative mass spectrometry," Molecular and Cellular Proteomics, vol. 8, no. 6, pp. 14361449, 2009.

[34] A. Supernat, A. Markiewicz, B. Seroczyńska et al., "CD73 expression as a potential marker of good prognosis in breast carcinoma," Applied Immunohistochemistry and Molecular Morphology, vol. 20, no. 2, pp. 103-107, 2012.

[35] S. Deaglio, K. M. Dwyer, W. Gao et al., "Adenosine generation catalyzed by CD39 and CD73 expressed on regulatory $\mathrm{T}$ cells mediates immune suppression," Journal of Experimental Medicine, vol. 204, no. 6, pp. 1257-1265, 2007.

[36] F. S. Regateiro, D. Howie, K. F. Nolan et al., "Generation of anti-inflammatory adenosine byleukocytes is regulated by TGF- $\beta$," European Journal of Immunology, vol. 41, no. 10, pp. 2955-2965, 2011.

[37] G. Borsellino, M. Kleinewietfeld, D. Di Mitri et al., "Expression of ectonucleotidase CD39 by Foxp3+ Treg cells: hydrolysis of extracellular ATP and immune suppression," Blood, vol. 110, no. 4, pp. 1225-1232, 2007.

[38] M. Mandapathil, B. Hilldorfer, M. J. Szczepanski et al., "Generation and accumulation of immunosuppressive adenosine by human CD4+CD25 high FOXP3+ regulatory T Cells," Journal of Biological Chemistry, vol. 285, no. 10, pp. 7176-7186, 2010.

[39] S. P. Hilchey, J. J. Kobie, M. R. Cochran et al., "Human follicular lymphoma CD39+-infiltrating T cells contribute to adenosine-mediated T cell hyporesponsiveness," Journal of Immunology, vol. 183, no. 10, pp. 6157-6166, 2009.

[40] K. M. Dwyer, D. Hanidziar, P. Putheti et al., "Expression of CD39 by human peripheral blood CD4+CD25+ T cells denotes a regulatory memory phenotype," American Journal of Transplantation, vol. 10, no. 11, pp. 2410-2420, 2010.

[41] M. Romio, B. Reinbeck, S. Bongardt, S. Hüls, S. Burghoff, and J. Schrader, "Extracellular purine metabolism and signaling of CD73-derived adenosine in murine treg and teff cells," American Journal of Physiology, vol. 301, no. 2, pp. C530-C539, 2011.

[42] F. Chalmin, G. Mignot, M. Bruchard et al., "Stat3 and Gfi1 transcription factors control Th17 cell immunosuppressive activity via the regulation of ectonucleotidase expression," Immunity, vol. 36, no. 3, pp. 362-373, 2012.

[43] P. E. Zarek, C. T. Huang, E. R. Lutz et al., "A2A receptor signaling promotes peripheral tolerance by inducing T-cell anergy and the generation of adaptive regulatory T cells," Blood, vol. 111, no. 1, pp. 251-259, 2008.

[44] J. Stagg, U. Divisekera, H. Duret et al., "CD73-deficient mice have increased antitumor immunity and are resistant to experimental metastasis," Cancer Research, vol. 71, no. 8, pp. 2892-2900, 2011.

[45] L. Wang, J. Fan, L. F. Thompson et al., "CD73 has distinct roles in nonhematopoietic and hematopoietic cells to promote tumor growth in mice," Journal of Clinical Investigation, vol. 121, no. 6, pp. 2371-2382, 2011.

[46] G. G. Yegutkin, F. Marttila-Ichihara, M. Karikoski et al., "Altered purinergic signaling in CD73-deficient mice inhibits tumor progression," European Journal of Immunology, vol. 41, no. 5, pp. 1231-1241, 2011.
[47] M. Mandapathil, M. J. Szczepanski, M. Szajnik et al., "Increased ectonucleotidase expression and activity in regulatory T cells of patients with head and neck cancer," Clinical Cancer Research, vol. 15, no. 20, pp. 6348-6357, 2009.

[48] M. Mandapathil, M. J. Szczepanski, M. Szajnik et al., "Adenosine and prostaglandin e2 cooperate in the suppression of immune responses mediated by adaptive regulatory T cells," Journal of Biological Chemistry, vol. 285, no. 36, pp. 2757127580, 2010.

[49] S. Jalkanen and M. Salmi, "VAP-1 and CD73, endothelial cell surface enzymes in leukocyte extravasation," Arteriosclerosis, Thrombosis, and Vascular Biology, vol. 28, no. 1, pp. 18-26, 2008.

[50] A. Ålgars, M. Karikoski, G. G. Yegutkin et al., "Different role of CD73 in leukocyte trafficking via blood and lymph vessels," Blood, vol. 117, no. 16, pp. 4387-4393, 2011.

[51] M. Takedachi, D. Qu, Y. Ebisuno et al., "CD73-generated adenosine restricts lymphocyte migration into draining lymph nodes," Journal of Immunology, vol. 180, no. 9, pp. 6288-6296, 2008.

[52] A. Zernecke, K. Bidzhekov, B. Özüyaman et al., "CD73/Ecto5 -nucleotidase protects against vascular inflammation and neointima formation," Circulation, vol. 113, no. 17, pp. 2120 2127, 2006.

[53] J. H. Mills, L. F. Thompson, C. Mueller et al., "CD73 is required for efficient entry of lymphocytes into the central nervous system during experimental autoimmune encephalomyelitis," Proceedings of the National Academy of Sciences of the United States of America, vol. 105, no. 27, pp. 9325-9330, 2008.

[54] C. S. Hilaire, C. Shira, S. G. Ziegler et al., "NT5E mutations and arterial calcifications," New England Journal of Medicine, vol. 364, no. 5, pp. 432-442, 2011.

[55] H. K. Eltzschig, L. F. Thompson, J. Karhausen et al., "Endogenous adenosine produced during hypoxia attenuates neutrophil accumulation: coordination by extracellular nucleotide metabolism," Blood, vol. 104, no. 13, pp. 3986-3992, 2004.

[56] P. F. Lennon, C. T. Taylor, G. L. Stahl, and S. P. Colgan, "Neutrophil-derived 5'-adenosine monophosphate promotes endothelial barrier function via CD73-mediated conversion to adenosine and endothelial A(2B) receptor activation," Journal of Experimental Medicine, vol. 188, no. 8, pp. 1433-1443, 1998.

[57] T. Kong, K. A. Westerman, M. Faigle, H. K. Eltzschig, and S. P. Colgan, "HIF-dependent induction of adenosine A2B receptor in hypoxia," FASEB Journal, vol. 20, no. 13, pp. 2242-2250, 2006.

[58] S. Ryzhov, J. L. McCaleb, A. E. Goldstein, I. Biaggioni, and I. Feoktistov, "Role of adenosine receptors in the regulation of angiogenic factors and neovascularization in hypoxia," Journal of Pharmacology and Experimental Therapeutics, vol. 320, no. 2, pp. 565-572, 2007.

[59] I. Feoktistov, A. E. Goldstein, S. Ryzhov et al., "Differential expression of adenosine receptors in human endothelial cells: role of A2B receptors in angiogenic factor regulation," Circulation Research, vol. 90, no. 5, pp. 531-538, 2002.

[60] A. Ahmad, S. Ahmad, L. Glover et al., "Adenosine A2A receptor is a unique angiogenic target of HIF- $2 \alpha$ in pulmonary endothelial cells," Proceedings of the National Academy of Sciences of the United States of America, vol. 106, no. 26, pp. 10684-10689, 2009.

[61] A. Desai, C. Victor-Vega, S. Gadangi, M. C. Montesinos, C. C. Chu, and B. N. Cronstein, "Adenosine A2A receptor stimulation increases angiogenesis by down-regulating production of the antiangiogenic matrix protein thrombospondin 1," Molecular Pharmacology, vol. 67, no. 5, pp. 1406-1413, 2005. 
[62] N. S. Umapathy, Z. Fan, E. A. Zemskov, I. B. Alieva, S. M. Black, and A. D. Verin, "Molecular mechanisms involved in adenosine-induced endothelial cell barrier enhancement," Vascular Pharmacology, vol. 52, no. 5-6, pp. 199-206, 2010.

[63] S. Narravula, P. F. Lennon, B. U. Mueller, and S. P. Colgan, "Regulation of endothelial CD73 by adenosine: paracrine pathway for enhanced endothelial barrier function," Journal of Immunology, vol. 165, no. 9, pp. 5262-5268, 2000.

[64] J. Niemelä, T. Henttinen, G. G. Yegutkin et al., "IFN- $\alpha$ induced adenosine production on the endotheliumml: a mechanism mediated by CD73 (ecto- $5^{\prime}$-nucleotidase) upregulation," Journal of Immunology, vol. 172, no. 3, pp. 16461653, 2004.

[65] J. Niemelä, I. Ifergan, G. G. Yegutkin, S. Jalkanen, A. Prat, and L. Airas, "IFN- $\beta$ regulates CD73 and adenosine expression at the blood-brain barrier," European Journal of Immunology, vol. 38, no. 10, pp. 2718-2726, 2008.

[66] N. D. Khoa, M. C. Montesinos, A. J. Williams, M. Kelly, and B. N. Cronstein, "Th1 cytokines regulate adenosine receptors and their downstream signaling elements in human microvascular endothelial cells," Journal of Immunology, vol. 171, no. 8, pp. 3991-3998, 2003.

[67] K. Kalsi, C. Lawson, M. Dominguez, P. Taylor, M. H. Yacoub, and R. T. Smolenski, "Regulation of ecto-5' -nucleotidase by TNF- $\alpha$ in human endothelial cells," Molecular and Cellular Biochemistry, vol. 232, no. 1-2, pp. 113-119, 2002.

[68] L. Airas, J. Niemela, and S. Jalkanen, "CD73 engagement promotes lymphocyte binding to endothelial cells via a lymphocyte function-associated antigen-1-dependent mechanism," Journal of Immunology, vol. 165, no. 10, pp. 5411-5417, 2000.

[69] L. Airas, J. Niemelä, M. Salmi, T. Puurunen, D. J. Smith, and S. Jalkanen, "Differential regulation and function of CD73, a glycosyl- phosphatidylinositol-linked 70-kD adhesion molecule, on lymphocytes and endothelial cells," Journal of Cell Biology, vol. 136, no. 2, pp. 421-431, 1997.

[70] N. Sethi and Y. Kang, "Unravelling the complexity of metastasis—molecular understanding and targeted therapies," Nature Reviews Cancer, vol. 11, no. 10, pp. 735-748, 2011.

[71] H. Lee, E. C. K. Lin, L. Liu, and J. W. Smith, "Gene expression profiling of tumor xenografts: in vivo analysis of organspecific metastasis," International Journal of Cancer, vol. 107, no. 4, pp. 528-534, 2003.

[72] J. Stagg, P. A. Beavis, U. Divisekera et al., "CD73-deficient mice are resistant to carcinogenesis," Cancer Research, vol. 72, no. 9, pp. 2190-2196, 2012.

[73] J. Stagg, U. Divisekera, N. McLaughlin et al., "Anti-CD73 antibody therapy inhibits breast tumor growth and metastasis," Proceedings of the National Academy of Sciences of the United States of America, vol. 107, no. 4, pp. 1547-1552, 2010.

[74] C. L. Richard, E. Y. Tan, and J. Blay, "Adenosine upregulates CXCR4 and enhances the proliferative and migratory responses of human carcinoma cells to CXCL12/SDF-1 $\alpha$," International Journal of Cancer, vol. 119, no. 9, pp. 2044-2053, 2006.

[75] E. C. Woodhouse, D. F. Amanatullah, J. A. Schetz, L. A. Liotta, M. L. Stracke, and T. Clair, "Adenosine receptor mediates motility in human melanoma cells," Biochemical and Biophysical Research Communications, vol. 246, no. 3, pp. 888-894, 1998.

[76] P. Zhou, X. Zhi, T. Zhou et al., "Overexpression of ecto-5'nucleotidase (CD73) promotes T-47D human breast cancer cells invasion and adhesion to extracellular matrix," Cancer Biology and Therapy, vol. 6, no. 3, pp. 426-431, 2007.
[77] L. Wang, X. Zhou, T. Zhou et al., "Ecto-5'-nucleotidase promotes invasion, migration and adhesion of human breast cancer cells," Journal of Cancer Research and Clinical Oncology, vol. 134, no. 3, pp. 365-372, 2008.

[78] X. Zhi, S. Chen, P. Zhou et al., "RNA interference of ecto-5' nucleotidase (CD73) inhibits human breast cancer cell growth and invasion," Clinical and Experimental Metastasis, vol. 24, no. 6, pp. 439-448, 2007.

[79] B. M. Künzli, M. I. Bernlochner, S. Rath et al., "Impact of CD39 and purinergic signalling on the growth and metastasis of colorectal cancer," Purinergic Signalling, vol. 7, no. 2, pp. 231-241, 2011.

[80] A. Ohta, E. Gorelik, S. J. Prasad et al., "A2A adenosine receptor protects tumors from antitumor T cells," Proceedings of the National Academy of Sciences of the United States of America, vol. 103, no. 35, pp. 13132-13137, 2006.

[81] D. Jin, J. Fan, L. Wang et al., "CD73 on tumor cells impairs antitumor T-cell responses: a novel mechanism of tumorinduced immune suppression," Cancer Research, vol. 70, no. 6, pp. 2245-2255, 2010.

[82] X. Zhou, X. Zhi, P. Zhou et al., "Effects of ecto-5' -nucleotidase on human breast cancer cell growth in vitro and in vivo.," Oncology Reports, vol. 17, no. 6, pp. 1341-1346, 2007.

[83] F. Ghiringhelli, L. Apetoh, A. Tesniere et al., "Activation of the NLRP3 inflammasome in dendritic cells induces IL$1 \beta$-dependent adaptive immunity against tumors," Nature Medicine, vol. 15, no. 10, pp. 1170-1178, 2009.

[84] L. Aymeric, L. Apetoh, F. Ghiringhelli et al., "Tumor cell death and ATP release prime dendritic cells and efficient anticancer immunity," Cancer Research, vol. 70, no. 3, pp. 855-858, 2010. 

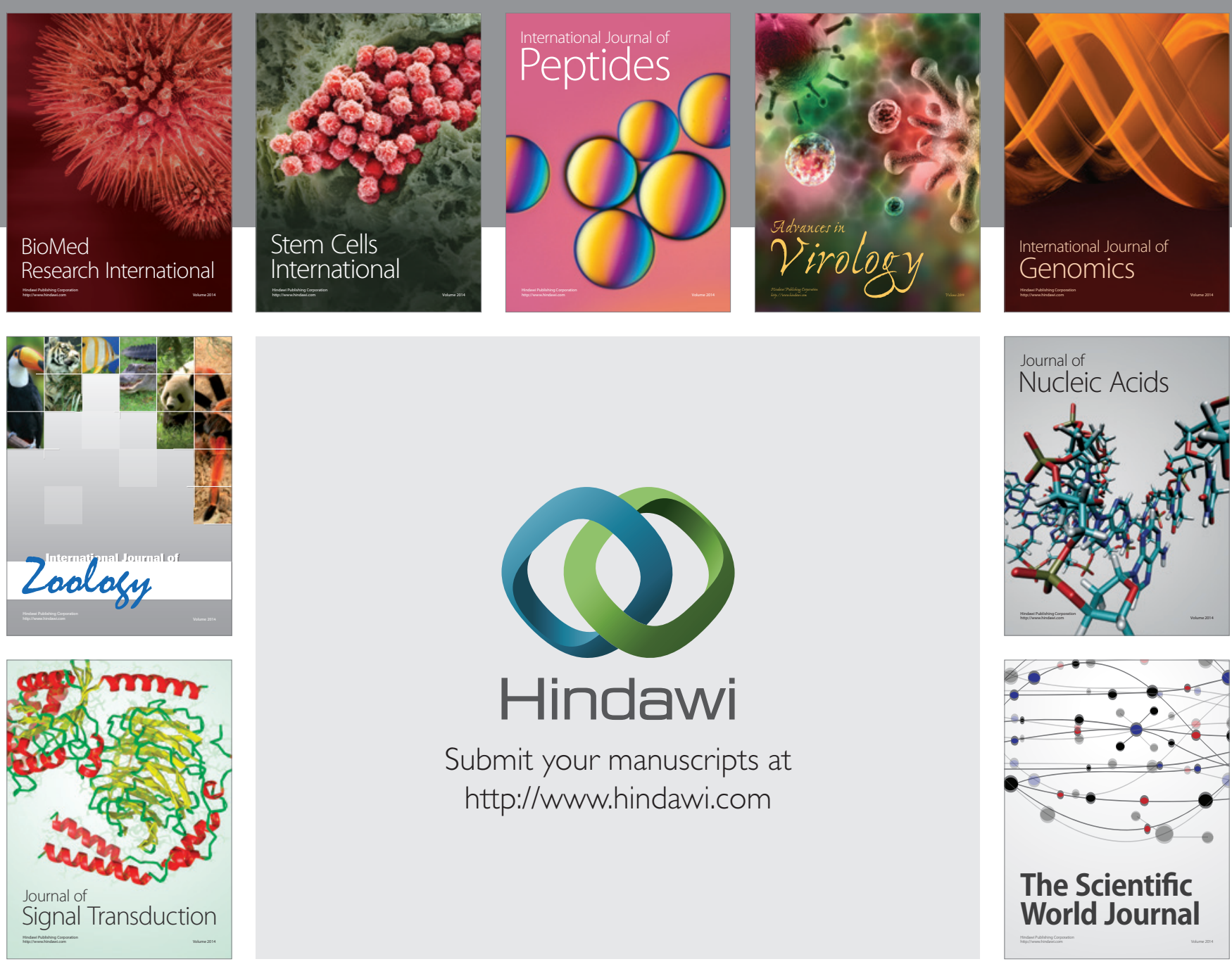

Submit your manuscripts at

http://www.hindawi.com
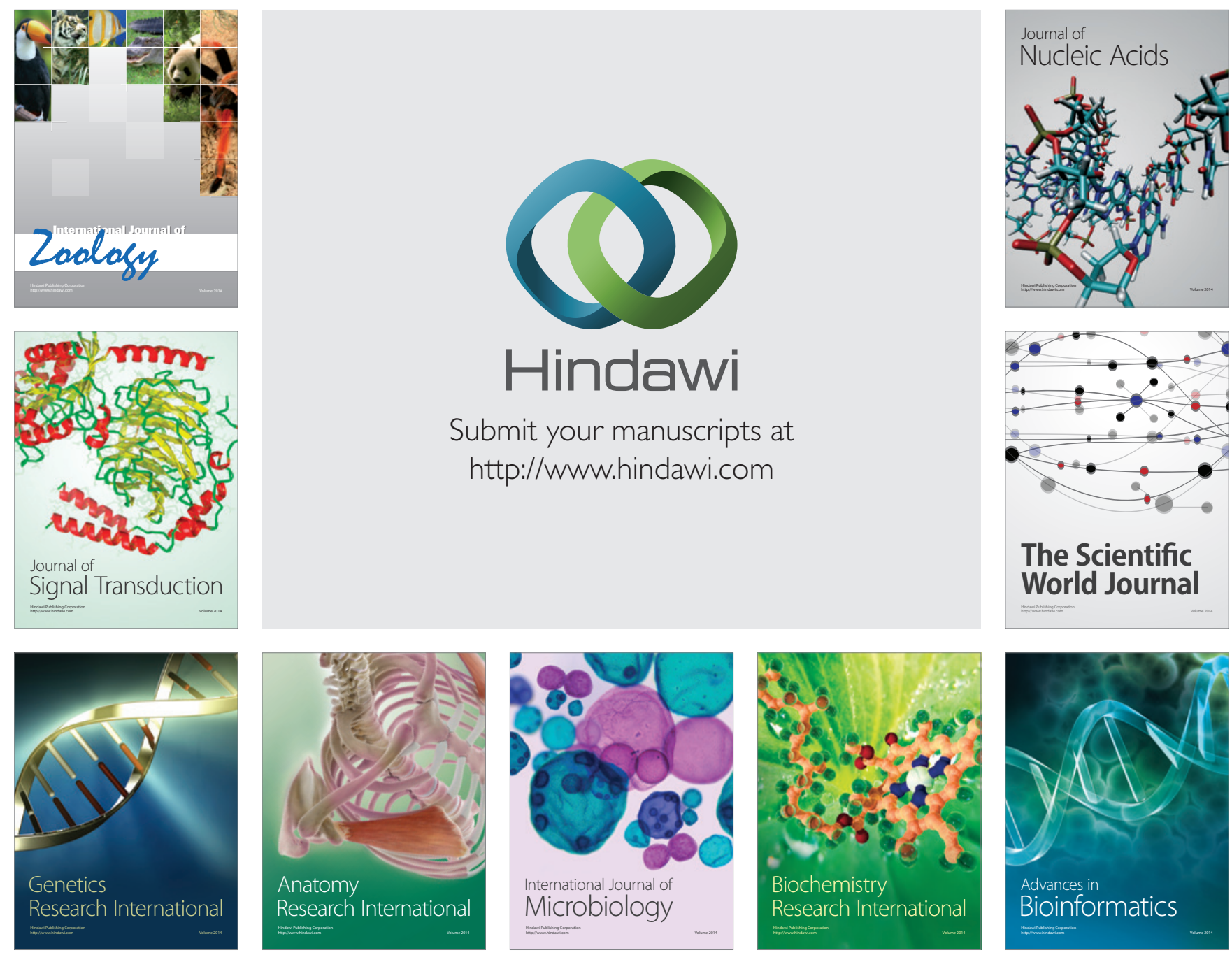

The Scientific World Journal
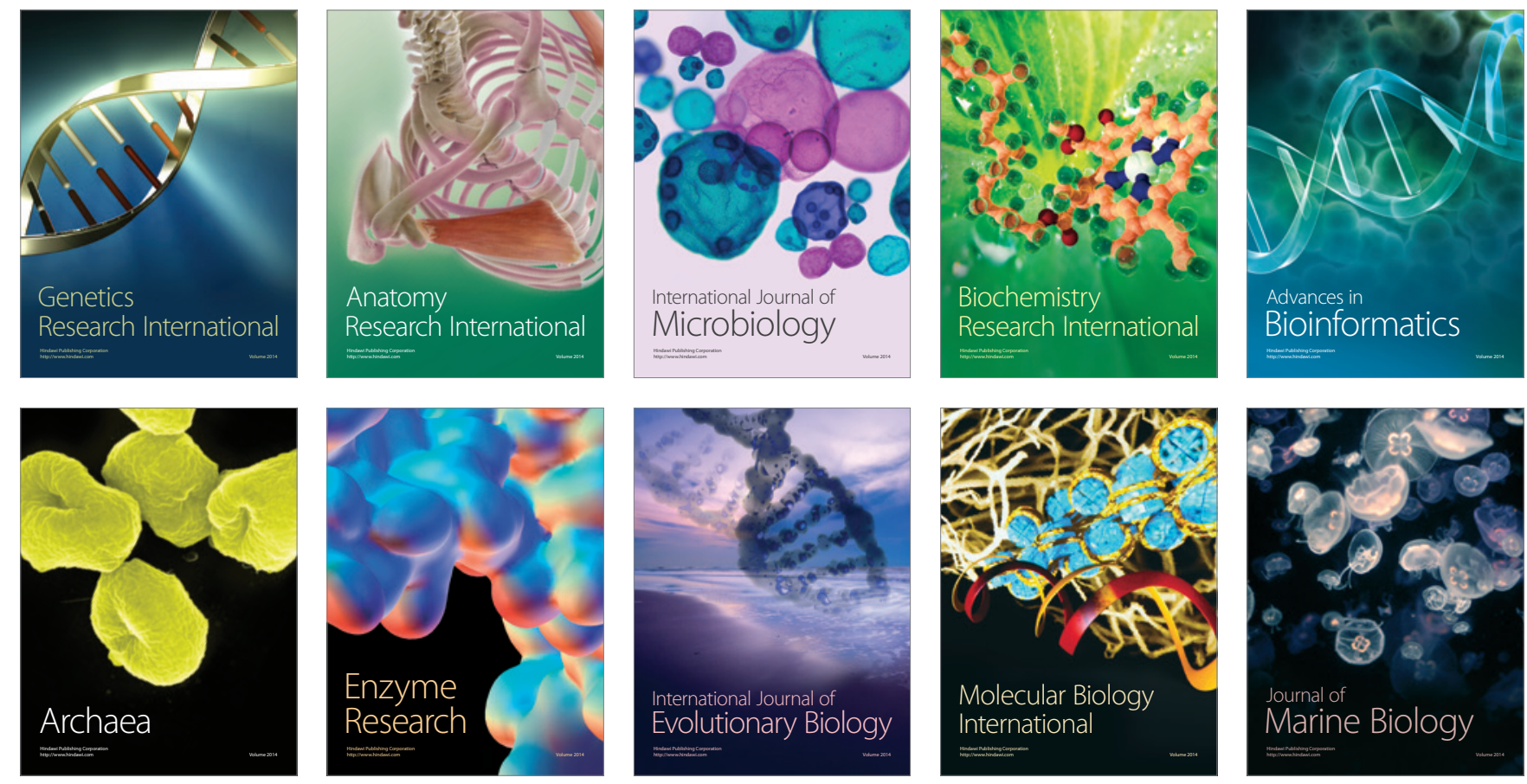